\title{
Vulner-abilité posthumaine
}

\section{Christine Daigle}

Correspondance: cdaigle@brocku.ca

Christine Daigle est professeure de philosophie, directrice du Posthumanism Research Institute et du Posthumanism Research Network, ainsi que Chancellor's Chair for Research Excellence à l'université Brock (St. Catharines, Ontario). Elle a beaucoup travaillé la pensée de Friedrich Nietzsche, Simone de Beauvoir, et Jean-Paul Sartre et a publié des articles et ouvrages sur divers aspects de leur philosophie. Sa recherche porte maintenant principalement sur la théorie posthumaniste, le féminisme matérialiste et la théorie des affects.

Résumé: Je propose de concevoir l'être humain comme transjectif : à la fois transsubjectif et transobjectif. Cela suppose de prendre en considération les enchevêtrements matériels dans lesquels nous existons. En prenant également en considération les concepts de transcorporéité et de créature bioculturelle offerts par Stacy Alaimo et Samantha Frost respectivement, nous sommes en mesure de bien comprendre les mécanismes qui sont au fondement de notre vulnérabilité. Puisque le féminisme matérialiste et la théorie des affects démontrent que notre relationalité est essentielle à notre devenir, je propose d'adopter le terme « affect—abilité » plutôt que « vulnérabilité » afin de nous éloigner des connotations négatives de ce dernier et de faciliter la reconnaissance et l'acceptation du sujet exposé, imprégné et imprégnant, que nous sommes.

Mots clés: Posthumanisme-Féminisme matérialiste-Vulnérabilité—Affects—Transcorporéité

Le posthumanisme critique, tel qu'articulé dans les travaux des féministes matérialistes, rejette la conception humaniste de l'être humain fondée sur deux dualismes : conscience/corps et humain/non-humain. En contrepartie, l'emphase est mise sur l'inextricable enchevêtrement de toutes créatures et sur l'inséparabilité de la conscience et du corps. Cela contribue à contrer l'exceptionnalisme humain et le point de vue anthropocentriste de l'humanisme. Le posthumanisme critique des féministes matérialistes tend à adopter une ontologie plate, dans laquelle il n'y a pas de hiérarchie. Rosi Braidotti, par exemple, nomme cela le "zoé-égalitarisme. » Ma notion de transjectivité s'inscrit dans ce courant et est en accord avec les concepts de transcorporéité, développé par Stacy Alaimo, et de créature bioculturelle, offerte par Samantha Frost. Je commence cet article par une brève discussion de ces concepts afin de lancer mon analyse de la vulnérabilité. J'argumenterai que celle-ci est potentiellement positive et générative et qu'il faut la reconcevoir comme un élément essentiel à notre épanouissement.

Je propose de concevoir l'être humain, ainsi que tous les autres êtres vivants, comme des êtres transjectifs : à la fois transsubjectifs et transobjectifs, enchevêtrés dans leurs expériences subjectives et dans leur matérialité. Il y a mouvement et enchevêtrement continu du subjectif à l'objectif, au matériel, à tel point que distinguer une pure subjectivité ou une pure objectivité devient impossible. ${ }^{2}$ Pour sa part, Stacy Alaimo adopte le terme de transcorporéité. Elle explique que nos corps sont faits de limites perméables_les muqueuses, la peau, les orifices_et que nous sommes littéralement des êtres poreux constitués par la matière qui nous entoure, nous habite, et passe en transit dans notre corps tout comme nous nous écoulons sans cesse dans cette matière, le transit allant dans les deux

\footnotetext{
${ }^{1}$ Voir son ouvrage The Posthuman.

2 J'ai commencé à développer ce concept dans l'essai «Trans-subjectivity/Trans-objectivity, » Feminist Phenomenology Futures. Un essai à paraitre développe plus avant la notion de transjectivité et la fonde dans un spinozisme deleuzien.
}

Daigle / Vulner-abilité posthumaine

doi:10.28984/ct.v2i1.267 
sens. Nous sommes ainsi des êtres toxiques et exposés: « always already penetrated by substances and forces that can never be properly accounted for » (Alaimo 5). Cela l'amène à dire que nous devons adopter un posthumanisme environnemental qui prenne en compte notre enchevêtrement matériel et corporel.

Comme l'être humain, et tous les êtres, sont en fluctuation constante de par leur exposition à la matière qui les traverse mais aussi dû à leurs interactions transsubjectives, il devient difficile d'établir un fondement pour l'agentivité. Cela est un problème important pour les féministes matérialistes pour qui un agent à qui on peut attribuer une responsabilité éthique et politique est nécessaire. ${ }^{3}$ L'ouvrage récent de Samantha Frost explore les mécanismes biochimiques qui nous régulent afin d'expliquer comment il est possible d'établir une agentivité sur les bases de ceux-ci, même si cet agent demeure un agent minimaliste. Elle explique que l'organisme est pénétré par son habitat et que celui-ci dépend de la circulation des atomes, molécules et cellules à travers ses nombreuses membranes pour sa subsistance, afin que la vie se poursuive et soit florissante. Elle dit que l'organisme « composes and recomposes itself continuously in response to and through engagement with its habitat. » (Frost 145). Parler d'un « dehors » ou d'un « dedans » des corps est futile. Il n'y a pas de telle distinction.

L'individu qui ressort de ceci est en fait un dividu en devenir constant. Nous sommes imprégnés du monde dans lequel nous sommes tout autant que nous l'imprégnons. Tous les êtres sont enchevêtrés et tous les êtres sont vulnérables. C'est la perméabilité des êtres qui les rend vulnérables. Toutes les expériences vécues par l'être transjectif sont transformatives, que ce soit avec des humains, des non-humains, des êtres animés ou pas, le monde dans son ensemble, les concepts, la culture, etc. Cette perméabilité nous rend vulnérables mais cela ne doit pas être compris de manière négative. Cette vulnérabilité peut générer un nouveau type de responsabilité éthique qui peut mener à un foisonnement de la vie sous toutes ses formes. ${ }^{4}$ En fait, cette vulnérabilité est nécessaire et doit être célébrée.

Judith Butler a beaucoup écrit sur la notion de vulnérabilité et la question de savoir qui compte moralement et qui « mérite d'être pleuré. ${ }^{5}$ C'est une question politique importante par exemple lorsque l'on pose la question des migrants : est-ce que les migrants font partie de notre cercle de préoccupation morale? Doit-on en prendre soin? Notre soin, notre compassion, notre action leur sont-ils dûs? Les gens les plus vulnérables sont souvent exclus de notre champ de considération morale ce qui les rend encore plus vulnérables. Butler examine ces questions et dit « the ontology of the body serves as a point of departure for ... a rethinking of responsibility ... precisely because, in its surface and its depth, the body is a social phenomenon; it is exposed to others, vulnerable by definition. » (Butler, Frames of War, 33). Mais l'être transjectif est encore plus vulnérable que ce que suggère Butler parce que sa perméabilité réside en son cœur biochimique: un être dont la survie et la vie florissante dépend du transit d'autres êtres à travers son être. La vulnérabilité posthumaine est non seulement potentiellement positive mais, plus encore, essentielle.

\footnotetext{
${ }^{3}$ Cela est particulièrement le cas chez les posthumanistes féministes qui adoptent cette philosophie en partie afin de se libérer des structures d'oppression qui émergent de l'humanisme exceptionnaliste. En lutte contre les systèmes d'oppression quant au sexe, à la race, à l'orientation sexuelle, etc. celles-ci ont besoin d'un agent qui puisse être visé comme responsable mais aussi d'un agent qui puisse lutter pour ses droits. Ce problème est complexe et des penseurs comme Braidotti proposent un essentialisme stratégique pour s'y attaquer, ce qui n'est pas sans susciter la controverse. ${ }^{4}$ Il n'y a pas place ici pour élaborer sur la responsabilité éthique et quelle forme celle-ci pourrait prendre. Cela devra faire l'objet de futurs travaux. Je peux toutefois indiquer qu'au fondement de cette responsabilité se trouve le désir de voir les êtres mener une vie enchevêtrée florissante.

${ }^{5}$ Elle pose la question « Whose life is grievable? » dans Frames of War et Precarious Life.
}

Daigle / Vulner-abilité posthumaine 
Comment doit-on comprendre cela? Le latin «vulner » signifie « blesser », « affecter », plus souvent de façon négative. Le sens commun que l'on donne au terme «vulnérabilité » est celui d'être susceptible d'être blessé physiquement ou émotionnellement. Cette susceptibilité génère un devoir pour les autres agents de ne pas abuser de cette vulnérabilité, voire même de protéger l'être vulnérable. Mais cette façon de concevoir la vulnérabilité présuppose que l'on considère le corps comme étant bien délimité et que l'on considère aussi qu'il faille maintenir et protéger ces limites. Toutefois, comme on l'a vu, on ne peut appliquer cela à l'être transjectif qui est un être transcorporel, exposé et perméable. Il faut plutôt prendre le terme de vulner-abilité de façon différente. L'être transjectif est vulner-able, ou plutôt vulner-apte, puisque c'est un corps qui fait et défait ce avec quoi il interagit. C'est un être qui est apte à «blesser», à affecter. Mais en étant empêtré dans l'enchevêtrement matériel dont on a parlé, l'être transjectif est à la fois apte à blesser et à être blessé. Jane Bennett dit que « in a knotted world of vibrant matter, to harm one section of the web may very well be to harm oneself. » (Bennett 13). Blesser, c'est se blesser et, se blesser, c'est aussi blesser. Nous ne sommes pas des êtres fermés sur soi qui interagissent les uns avec les autres. Toute interaction est un transit, un enchevêtrement, une constitution et une destruction du « soi ». L'« abilité » dont il est question ici, l'aptitude à blesser, n'est toutefois pas l'expression d'une agentivité intentionnelle forte. Cette aptitude est celle d'un être qui est lui-même le fruit d'un enchevêtrement de relations et d'êtres qui le traversent. Notre aptitude à blesser est donc souvent une direction sans intention, c'est à dire un acte d'agentivité mais sans intentionalité directe ou forte. ${ }^{6}$

Nous cherchons, la plupart du temps, à nous protéger et à réduire notre vulnérabilité. Or, c'est mal comprendre le type de créature que nous sommes. Simone Drichel dit quelque chose de très intéressant à cet égard: «In seeking to defend ourselves, we-perversely—come to violate ourselves, or, to put this differently, what we preserve in 'self-preservation' is what makes the self 'inhuman' rather than human » (Drichel 22). En faisant l'effort de nous protéger et de nous rendre invulnérables, nous nous faisons violence et nous nous dés-humanisons. Il serait préférable d'accepter notre vulner — abilité et de chercher à vivre une multitude d'expériences tout en reconnaissant que certaines d'entre elles seront peut-être transformatives de façon inconfortable ou carrément destructive. L'important est de cesser de se concevoir comme tout-puissants ou, en tout cas, d'essayer de l'être en nous rendant invulnérables. Cette tentative est futile de toute façon et, comme le dit Claire Colebrook, il s'agit d'un reste de pensée humaniste qui conçoit l'humain comme omnipotent. ${ }^{7}$ Reconnaître notre être transjectif et sa vulner-abilité fondamentale, reconnaître que nous dépendons de cette vulnérabilité sociale, matérielle et subjective afin de mener une vie florissante nécessite que nous abandonnions les idéaux humanistes dont nous avons souffert trop longtemps, nous et tous les autres êtres avec qui nous partageons le monde. Si toute expérience n'est pas bonne en soi, toute expérience constitue l'être transjectif.

On peut comprendre et choisir de maintenir cette vulner-abilité si nous comprenons qu'il y a plusieurs dimensions à notre relationalité et notre enchevêtrement. Comprendre cela, c'est aussi comprendre que nous n'avons jamais été l'humain de la pensée humaniste. C'est comprendre que les philosophies qui ont misé sur la raison, les dichotomies sujet/objet, pensée/corps et humain/nature,

\footnotetext{
${ }^{6}$ La notion de direction sans intention est amenée par Frost pour distinguer l'agentivité à l'œuvre dans les processus biochimiques de l'agentivité d'une personne, l'intentionalité telle qu'on la conçoit communément, qui est par ailleurs aussi animée par la multitude de directions sans intention qui la construit. Voir Biocultural Creatures. Il faut comprendre que même si elles sont distinctes ces deux types d'agentivité, la direction sans intention et l'agentivité intentionelle forte, sont toujours interreliées.

${ }^{7}$ Voir Colebrook 2014, 13.
}

Daigle / Vulner-abilité posthumaine

doi:10.28984/ct.v2i1.267 
ont introduit des distinctions et des limites qui, en fait, n'existent pas. Comprendre tout cela nous permet de redevenir le posthumain que nous avons toujours été-un ante-humain, soit l'humain d'avant l'humanisme. Cela nous permet aussi de vaincre l'exceptionnalisme humain si dommageable. Comprendre notre vulner-abilité comme étant le fondement de la vie florissante signifie que nous pouvons embrasser cette vulnérabilité et la chérir. Mais cela entraîne aussi des prises de position éthiques nécessaires.

Redécouvrir l'être posthumain que nous avons toujours été, l'être transjectif et sa constitution matérielle, nous offre une meilleure compréhension des multiples dimensions de la relationalité avec les êtres avec lesquels nous sommes toujours enchevêtrés. Nous pouvons aussi penser la vulner-abilité en termes génératifs. Stacy Alaimo nous invite à concrétiser notre exposition : nous sommes exposés et devons chercher à l'être. Elle veut que nous embrassions notre perméabilité. Elle dit :

... exposure entails the intuitive sense or the philosophical conviction that the impermeable Western human subject is no longer tenable. Performing exposure as an ethical and political act means ... to grapple with the particular entanglements of vulnerability and complicity that radiate from disasters and their terribly disjunctive connection to everyday life in the industrialized world. To occupy exposure as insurgent vulnerability is to perform material rather than abstract alliances (Alaimo 5).

Reconnaitre et accepter le sujet exposé qui est toujours imprégné et imprégnant implique un changement radical dans notre vision du monde, un devenir posthumain. Le terme vulner-abilité est peut-être inapproprié. Il possède un trop plein de bagage négatif qui nous porte instinctivement à nous en garder. Peut-être doit-on conserver ce terme seulement pour l'exposition négative. Ce n'est pas tant la blessure (vulner) qui est nécessaire mais l'affectivité (afficere). Cette capacité d'un être à en affecter un autre est à la fois positive et négative et est en fait nécessaire au développement de chacun. ${ }^{8}$ Remplacer vulner_abilité par affect—abilité est en quelque sorte un retour à Spinoza et sa philosophie des affects dont on ne peut se garder puisqu'ils sont l'essence de ce que nous sommes et de nos relations. C'est un mouvement nécessaire pour nous guérir de l'humanisme.

${ }^{8}$ Il est important de noter que les dimensions de la vulner—abilité dont j’ai parlées sont interreliées et agissent les unes sur les autres.

Daigle / Vulner-abilité posthumaine 


\section{Bibliographie}

Alaimo, Stacy. Exposed: Environmental Politics \& Pleasures in Posthuman Times. University of Minnesota Press, 2016.

Bennett, Jane. Vibrant Matter: A Political Ecology of Things. Duke University Press, 2010.

Braidotti, Rosi. The Posthuman. Polity, 2013.

Butler, Judith. Frames of War: When is Life Grievable? Verso, 2009.

. Precarious Life: The Powers of Mourning and Violence, Verso, 2006.

Colebrook, Claire. Death of the Posthuman: Essays on Extinction I. Open Humanities Press, 2014.

Daigle, Christine. «Trans-subjectivity/Trans-objectivity. » Feminist Phenomenology Futures. Edited by Helen Fielding and Dorothea Olkowskim. Indiana University Press, 2017, 183-199.

Drichel, Simone. « Reframing Vulnerability: ‘so obviously the problem...’? » SubStance, vol. 42, no. 3, 2013, pp. 3-27.

Frost, Samantha. Biocultural Creatures. Toward a New Theory of the Human. Duke University Press, 2016. 